\title{
Polymorphonuclear leucocyte motility in men with ankylosing spondylitis
}

\author{
C T PEASE, M FENNELL, AND D A BREWERTON \\ From the Department of Rheumatology, Westminster Hospital, London
}

SUMMARY The polymorphonuclear leucocyte (PMN) response to a chemotactic or chemokinetic stimulus is enhanced in men with ankylosing spondylitis (AS). This effect does not parallel the severity of disease activity or the size of the acute phase response, and it is independent of nonsteroidal anti-inflammatory drug treatment. Polymorph function is normal in HLA-B27 positive brothers of probands with AS and in other HLA-B27 positive individuals in the absence of disease. Polymorph motility is also normal in patients with psoriasis vulgaris or Crohn's disease, indicating that enhanced PMN motility is not a non-specific consequence of all inflammatory disorders.

Key words: chemotaxis.

Healthy individuals with HLA-B27 are more likely to develop ankylosing spondylitis (AS) than B27 negative subjects and are also prone to develop a reactive arthritis after exposure to certain bacteria. The fundamental mechanisms involved for susceptibility to these disorders are unknown.

Previously, it has been reported that the polymorphonuclear leucocyte (PMN) response to certain chemotactic substances is enhanced in men with AS, ${ }^{1}{ }^{2}$ HLA-B27 positive men with yersinia arthritis, ${ }^{34}$ and in healthy HLA-B27 positive controls. ${ }^{1-3}$ Thus polymorphs from HLA-B27 positive individuals may inherit an enhanced ability to respond to certain chemotactic stimuli. It has been proposed that such hyperresponsive PMNs would accumulate in large numbers at sites of inflammation and may be responsible for an excessive inflammatory response. ${ }^{5}$ Clinically, patients with either yersinia arthritis or sexually acquired reactive arthritis tend to have more severe disease if they are HLA-B27 positive. ${ }^{67}$ The situation is less clear with AS because of the difficulty in recognition and diagnosis of mild cases, but it is known that acute uveitis occurs less frequently in patients with AS who do not have B27. The purpose of this study was to investigate further the relation between PMN migration, HLA-B27, and AS.

Accepted for publication 2 June 1988.

Correspondence to Dr C T Pease, Department of Rheumatology, Charing Cross Hospital, Fulham Palace Road, London W6 8RF.

\section{Subjects and methods}

PATIENTS AND CONTROLS

Forty men with classical AS were investigated. Thirty seven were HLA-B27 positive, three B27 negative. Their ages ranged from 22 to 72 years (mean 43.5) and duration of disease ranged from three to 52 years (mean 19). Drug treatment was not discontinued before the investigation. At the time of testing no patient or control had symptoms or signs of an acute bacterial or viral infection.

Seventeen men with AS attending an outpatient clinic at Westminster Hospital had healthy brothers willing to participate. All of the probands and 10 of the 17 brothers were B27 positive. The probands and controls were examined, and the ranges of chest expansion and spinal movement recorded. Three of the probands with AS had HLA identical brothers, two of whom on examination were proved to have AS. Thus these two pairs were analysed separately. The remaining 15 healthy brothers had no symptoms to suggest AS or uveitis, and examination was normal. An $x$ ray examination of the spine was not performed routinely. Two brothers had been $x$ rayed previously; the films were reviewed and found to be normal. The mean age of the probands was 43 years (range 22-61) and of the healthy brothers 43 years (18-64). Each pair was tested concurrently with a control matched for age and sex. Haemoglobin $(\mathrm{Hb})$, erythrocyte sedimentation rate (ESR), and $C$ reactive protein (CRP) values differed 
significantly between probands and brothers. The probands had the following values: Hb 109-162 g/l (mean $146 \mathrm{~g} / \mathrm{l}$ ), ESR $2-49 \mathrm{~mm} / \mathrm{h}$ (mean $16 \mathrm{~mm} / \mathrm{h}$ ), CRP 6-50 mg/l (mean $15 \mathrm{mg} / \mathrm{l}$ ); and the values for the brothers were $\mathrm{Hb} 135-178 \mathrm{~g} / \mathrm{l}$ (mean $158 \mathrm{~g} / \mathrm{l}$ ), ESR $1-6 \mathrm{~mm} / \mathrm{h}$ (mean $2 \mathrm{~mm} / \mathrm{h}$ ), CRP-all values below $6 \mathrm{mg} / \mathrm{l}$. Ten healthy HLA-B27 positive individuals with neither history nor signs of AS or family history of AS were used as an additional control group for the brother study.

The B27 negative controls were members of staff of St Stephen's and Westminster Hospitals. They were selected using the following criteria: male, aged between 20 and 60 years, no joint disease, no family history of AS, and no current drug treatment. All subjects were tissue typed, and only those without HLA-B27 were included in the investigation.

\section{DISEASE ACTIVITY}

Each subject's disease activity was assessed at every visit. The amount of back pain was recorded on a four point scale; peripheral joint involvement was noted and graded on a similar scale; the duration of early morning stiffness was recorded in minutes; and the need for drug treatment and its effectiveness were recorded. From this data, and from previous knowledge of the patient, the activity of disease was classified in three groups: 1. Active-Significant trunk pain or active peripheral arthritis with morning stiffness in excess of one hour. Regular drug treatment required. 2. Probably active-Patients in whom an acute episode had settled incompletely or in whom significant morning stiffness worsened if drug treatment was discontinued. 3. InactiveSymptomless, or mild back pain with less than 20 minutes' morning stiffness. Drugs taken irregularly or not at all.

Blood was taken at each visit for ESR and CRP estimations, but these values were not used in the clinical assessment of disease activity.

\section{METHODS}

Polymorphonuclear leucocytes were separated from heparinised blood by a Hypaque-Ficoll separation procedure. ${ }^{9}$ Cells were washed three times with minimum essential medium (MEM) supplemented with $1 \%$ albumin and were then resuspended in MEM at a concentration of $3 \cdot 2 \times 10^{7} \mathrm{PMNs} / \mathrm{ml}$. After the final wash $P M N$ viability was approximately $\mathbf{9 8 \%}$ when measured by trypan blue dye exclusion.

\section{Agarose plate method}

The procedure followed that described by Repo. ${ }^{10}$ Two wells were cut $2.5 \mathrm{~mm}$ apart in an agarose gel with a template and a $3 \mathrm{~mm}$ skin biopsy punch (Steifel Lab (UK), Slough, UK). To assess unstimu- lated migration $2.5 \times 10^{-3} \mathrm{PMNs}$ suspended in $\mathrm{MEM}_{\vec{\prime}}^{\Rightarrow}$ were placed in one well and MEM in the opposite well. To measure directed PMN migration $7 \mu \mathrm{l} N-\mathrm{O}$ formyl-methionyl-leucyl-phenylalanine (FMLP) at a흔 concentration of $10^{-7} \mathrm{~mol} / 1$ or $7 \mu \mathrm{l}$ zymosan activated $\frac{\bar{m}}{\bar{\sigma}}$ pooled serum $(\mathrm{ZAS}=90 \%$ serum +zymosan suspen- $\stackrel{\mathbb{\Phi}}{\Omega}$ sion $10 \mathrm{mg} / \mathrm{ml}$ ) was placed opposite the cell well. Plates were incubated in a humidified incubator at ${ }_{-}^{\infty}$ $37^{\circ} \mathrm{C}$ for two and a half hours and then the plates. were fixed and stained. The leading two cell distance $\vec{\omega}$ was measured. Each experiment was performed in triplicate.

\section{Microdroplet technique}

The microdroplet technique for measuring PMN $\vec{\omega}$ motility has been described by McCoy at al. ${ }^{110}$ Polymorphs obtained from heparinised venous blood were suspended in MEM at a concentration of $\vec{c}_{\vec{c}}$ $3.2 \times 10^{7} \mathrm{PMN} / \mathrm{ml}$ of MEM. Agarose (Marine Colloids Inc) at a concentration of $0.8 \%$ w/v was autoclaved, and while still molten, placed in a water bath at $37^{\circ} \mathrm{C}$. An equivalent volume of double $\vec{\bullet}$ strength $\mathrm{MEM}+1 \%$ human serum albumin was added to the agarose. Equal volumes of the agarose-MEM mixture and of the PMN suspension wereo mixed and $2 \mu \mathrm{l}$ droplets of the mixture placed in the centre of a cooled 96 well microtitre plate (non-o tissue cultured-Sterilin). The droplets required $\mathrm{a} \frac{\mathrm{O}}{\odot}$ few minutes to solidify, after which $100 \mu$ l of MEM음 or the test substance (FMLP/serum/ZAS) was care- $\overrightarrow{\overrightarrow{0}}$ fully added to each well. Use of a 96 well microtitre 3 plate meant that five concentrations of each sub-? stance could be tested simultaneously. The plates. were incubated at $37^{\circ} \mathrm{C}$ for three hours and then transferred to a fridge at $4^{\circ} \mathrm{C}$ until read. Cell ${ }^{\circ}$ migration was assessed by measuring the distance of the outermost cell from the edge of the droplet in four directions, each experiment being performed in duplicate.

S T A T IS TICS
The data were analysed by the Wilcoxon rank sum test.

\section{Results}

POLYMORPH MOTILITY IN AS

Blood was obtained from 30 HLA-B27 positive men with AS and 30 B27 negative controls matched for ${ }^{\text {? }}$ age and sex, and their PMN motility was compared using the agarose plate techniques. The results for unstimulated polymorph motility (random) were similar both for men with AS and control subjects. Directed polymorph motility, stimulated by FMLPO or ZAS, was significantly increased in patients with 
AS compared with the control group (Wilcoxon $\mathrm{p}<0.001$ and $\mathrm{p}<0.02$ respectively). The mean (SD) results obtained for men with AS were unstimulated migration $1.02(0.32) \mathrm{mm}$; FMLP $2.23(0.43) \mathrm{mm}$; ZAS $1.56(0.4) \mathrm{mm}$. The results for control subjects were unstimulated migration $0.93(0.24) \mathrm{mm}$; FMLP $1.95(0.5) \mathrm{mm}$; ZAS $1.41(0.31) \mathrm{mm}$.

Assessment of PMN migration towards FMLP, by use of the chemotactic differential (directed motility minus unstimulated motility), gave similar results (Wilcoxon $\mathrm{p}=\mathbf{0} \cdot 001$ ). Polymorph motility results for the three B27 negative patients with AS were similar to those obtained for the B27 positive patients (results not presented).

\section{EFFECT OF AS DISEASE ACTIVITY ON PMN MOTILITY}

Polymorph motility results, obtained using the agarose plate technique, for 20 patients with active AS were compared with those obtained for 18 patients with clinically inactive AS. No significant difference in PMN motility was recorded for unstimulated migration or for the response to ZAS or FMLP. Yet when the results for patients with either active AS or inactive AS were compared with the results for the controls, who had been tested concurrently, a significant difference was observed in response to either ZAS $(\mathrm{p}<0.05, \mathrm{p}<0.05$ respectively) or FMLP ( $<<0.02, p=0.001$ respectively). The mean (SD) results were unstimulated migration -active AS 1.02 (0.36) mm, control $0.90(0.25) \mathrm{mm}$; inactive AS 0.89 (0.19) $\mathrm{mm}$, control $0.82(0.18) \mathrm{mm}$. ZAS results-active AS $1.52(0.44) \mathrm{mm}$, control $1.32(0.33) \mathrm{mm}$; inactive AS $1.5(0.46) \mathrm{mm}$, control $1.33(0.35) \mathrm{mm}$. Figure 1 shows the results for FMLP.

Retrospectively, the agarose plate results for each patient with AS were matched with their CRP results obtained on the same day. This enabled us to compared the PMN response to FMLP from 10 patients with active AS and high CRP concentrations and 10 patients with inactive AS and normal CRP concentrations. No correlation could be observed between the CRP concentration and PMN reponses to FMLP (data not illustrated).

POLYMORPH MOTILITY IN RELATIONTO DRUG TRE ATM EN T

To assess whether non-steroidal anti-inflammatory drugs taken orally could influence PMN motility in vitro PMN migration was measured using the agarose plate technique before and immediately after a two week course of naproxen $(500 \mathrm{mg}$ twice a day) in 11 men with AS. No drugs, except paracetamol, were permitted for one week before the drug trial. In men with AS both unstimulated and

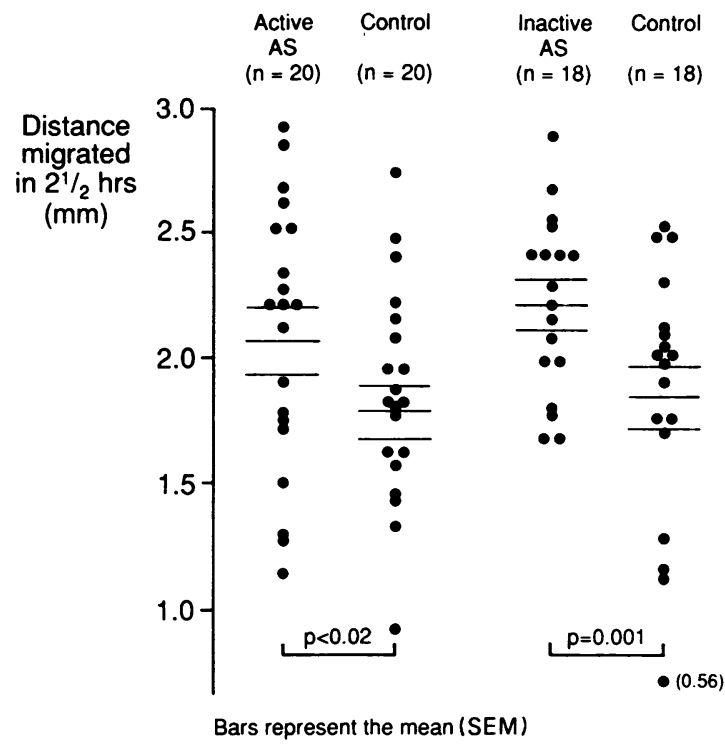

Fig. 1 Polymorph motility results for men with active or inactive ankylosing spondylitis (AS) compared with results obtained for the B27 negative controls. The method used was the agarose plate technique and the chemoattractant was $N$-formyl-methionyl-leucyl-phenylalanine $\left(10^{-7}\right.$ molll $)$.

directed polymorph motility were unaffected by two weeks of oral naproxen. The results for the control population, who had been tested in parallel with the AS group, but who had not received nonsteroidal anti-inflammatory drug treatment, were similar on both occasions.

Baseline results (mean (SD)): unstimulated migration-AS $0.95(0.18) \mathrm{mm}$, control 1.03 $(0.26) \mathrm{mm}$; FMLP-AS $2.23(0.32) \mathrm{mm}$, control $2.12(0.45) \mathrm{mm}$; ZAS -AS $1.47(0.32) \mathrm{mm}$, control $1.51(0.23) \mathrm{mm}$. Results after two weeks: unstimulated migration-AS $0.98(0.18) \mathrm{mm}$, control 0.9 $(0.2) \mathrm{mm}$; FMLP - AS 2.08 (0.27) $\mathrm{mm}$, control 1.88 $(0.29) \mathrm{mm}$; ZAS-AS $1.35(0.34) \mathrm{mm}$, control 1.36 $(0 \cdot 36) \mathrm{mm}$.

\section{THE BROTHER STUDY}

The agarose plate technique was used to assess PMN motility in 15 HLA-B27 positive men with AS, their healthy brothers (eight HLA-B27 positive), 15 unrelated B27 negative controls, and, in addition, 20 other controls, of whom half were B27 positive.

Unstimulated PMN motility was similar in all groups. The mean (SD) results for men with AS were $0.86(0.22) \mathrm{mm}$, healthy brothers 0.79 
$(0.23) \mathrm{mm}, \mathrm{B} 27$ negative controls $0.84(0.22) \mathrm{mm}$, and in the second control group of $10 \mathrm{~B} 27$ positive controls $0.63(0.14) \mathrm{mm}$ and $10 \mathrm{~B} 27$ negative controls $0.66(0.21) \mathrm{mm}$.

Figure 2 shows the results obtained for migration towards FMLP $\left(10^{-7} \mathrm{~mol} / \mathrm{l}\right)$. A significant difference was noted between the probands with AS and the group of 15 brothers (Wilcoxon $p<0.02$ ). No statistical difference was found to exist between the B27 positive or B27 negative brothers or controls. The results for PMN migration towards ZAS were similar, with a significant difference noted between the probands with AS and the 15 brothers (Wilcoxon $p=0.003$ ). The mean (SD) results for migration to ZAS were AS men $1.48(0.43) \mathrm{mm}$, brothers 1.24 $(0.4) \mathrm{mm}, \mathrm{B} 27$ negative controls $1.28(0.37) \mathrm{mm}$, and in the second control group B27 positive-

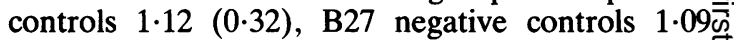
$(0 \cdot 28) \mathrm{mm}$.

\section{SERUM EXPERIMENTS}

\section{Preincubation of PMNs with serum}

Polymorphs from nine patients with AS and nine controls were preincubated for 30 minutes with MEM, $10 \%$ AS serum, or control serum before the. standard agarose plate chemotaxis assay. Unstimulated PMN motility was not affected by preincubation with serum (Table 1). Polymorph migration towardse either FMLP or ZAS was depressed after preincubation of PMNs in serum (either homologous orpo autologous) when compared with the results obtained after preincubation in MEM alone. Preincubation of

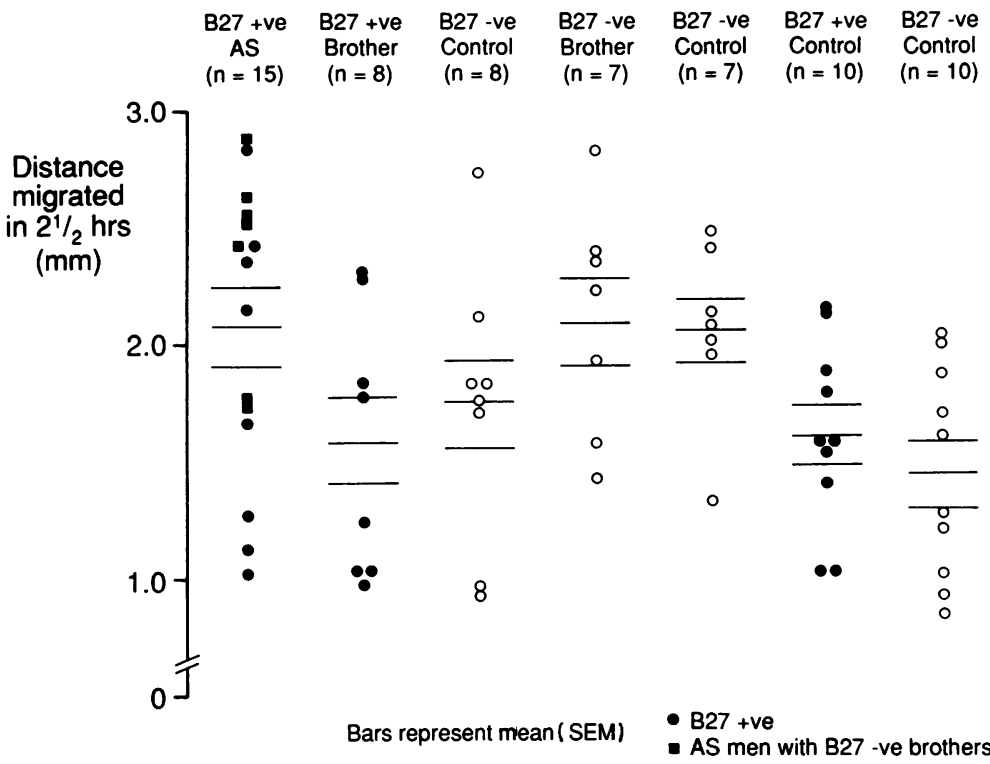

Fig. 2 Polymorph motility results formen with ankylosing spondylitis (AS), their healthy brothers (HLA B27 positive and B27 negative), and B27 positive controls compared with the results obtained for $B 27$ negative controls matched for age and sex. The method used was the agarose plate technique and the chemoattractant was $N$-formyl-methionyl-leucylphenylalanine $\left(10^{-7}\right.$ molll $)$.

Table 1 Effect on polymorph migration of preincubation for 30 minutes in $10 \%$ control serum or $10 \%$ serum from men $N$ with ankylosing spondylitis and comparison with the results of preincubation in minimum essential medium alone. ${ }^{*}$ Results - . are mean $(S D)$

\begin{tabular}{|c|c|c|c|c|c|c|}
\hline & \multicolumn{3}{|c|}{$\begin{array}{l}\text { AS PMNst ( } n=9) \\
\text { preincubation with: }\end{array}$} & \multicolumn{3}{|c|}{$\begin{array}{l}\text { Control PMNs }(n=9) \\
\text { preincubation with: }\end{array}$} \\
\hline & $M E M \dagger$ & $A S$ serum & Control serum & $M E M$ & $A S$ serum & Control serum \\
\hline Unstimulated migration (mm) & $0.82(0 \cdot 15)$ & $0 \cdot 88(0 \cdot 3)$ & $0.92(0.35)$ & $0.87(0 \cdot 28)$ & $0.82(0 \cdot 33)$ & $0.80(0.33)$ \\
\hline $\begin{array}{l}\text { Zymosan activated sera } \\
\text { (90\% sera+zymosan) (mm) }\end{array}$ & $1.47(0.4)$ & $1.29(0.38)$ & $1.29(0.48)$ & $1.35(0.42)$ & $1.04(0.42)$ & $1 \cdot 2(0.47)$ \\
\hline FMLP $\dagger\left(10^{-7} \mathrm{~mol} / \mathrm{l}\right)(\mathrm{mm})$ & $2 \cdot 12(0.54)$ & $1.87(0.63)$ & $1.81(0.72)$ & $1.85(0.69)$ & $1.66(0.67)$ & $1.6(0 \cdot 69)$ \\
\hline
\end{tabular}

*The polymorph motility was measured by the agarose plate technique. $\dagger \mathrm{AS}=$ ankylosing spondylitis; PMNs=polymorphonuclear leucocytes; MEM=minimum essential medium; FMLP=N-formyl-methionyl leucyl-phenylalanine. 


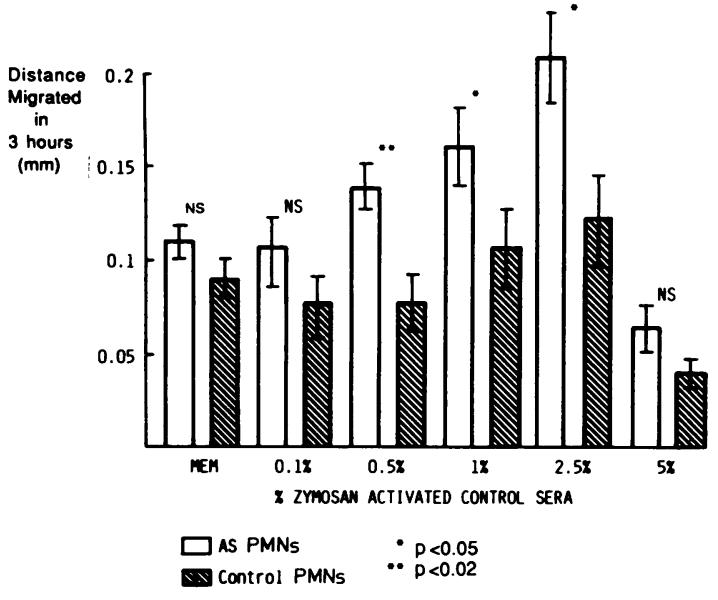

(each column represents the means for 11 individuals (SEM) )

Fig. 3 Comparison of the results obtained with the microdroplet technique for polymorphs from men with ankylosing spondylitis (AS) and from B27 negative controls. Zymosan activated control serum was used as the chemoattractant. $M E M=$ minimum essential medium.

control PMNs in AS sera or control sera did not enhance the PMN response to either FMLP or ZAS.

\section{Chemokinetic PMN motility in patients with $A S$ and in controls}

The PMN response to different uniform concentrations of serum, ZAS, or FMLP was studied in 11 men with $\mathrm{AS}$ and 11 controls using the microdroplet technique. When unactivated serum was used in the supernate AS cells were more motile than control PMNs, but only at a $1 \%$ serum dilution $(p<0.05)$ (date not illustrated). This result was, therefore, of doubtful significance. Figure 3 illustrates the results for zymosan activated sera. The distances migrated by AS cells were significantly greater than control PMNs for several different concentrations of ZAS. But the AS and control PMN responses to seven different concentrations of FMLP were identical (Fig. 4).

\section{Discussion}

This study has shown that the PMN response to two chemotactic substances is enhanced in men with AS. In contrast with earlier reports, ${ }^{1-3}$ this exaggerated PMN response was not found in healthy B27 positive brothers of men with AS or in a second

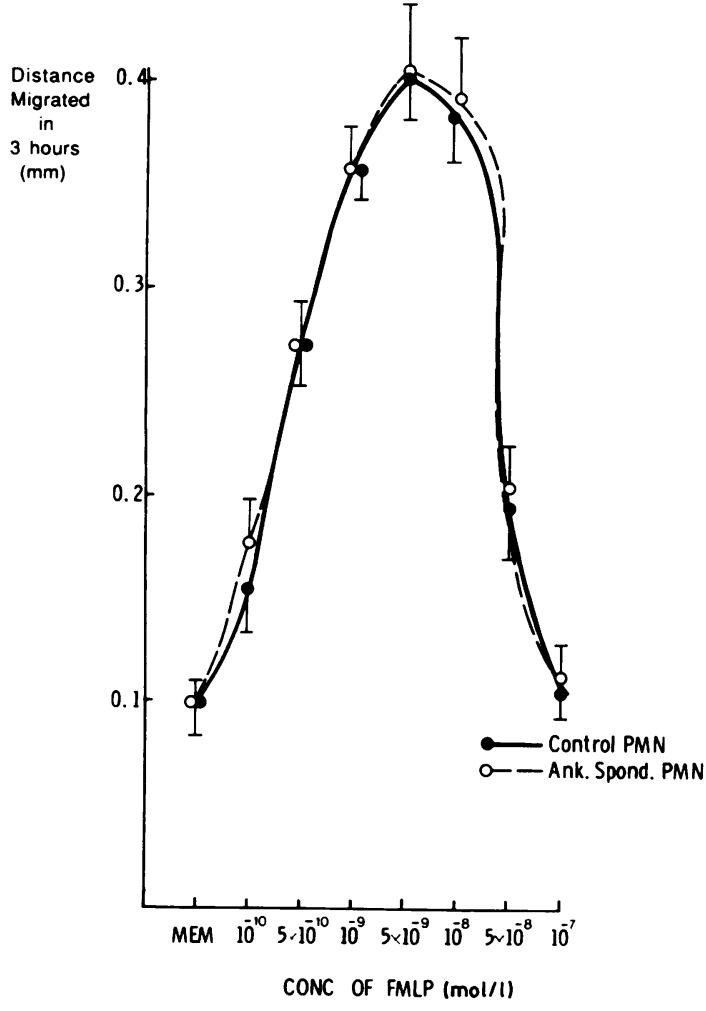

(each point represents the mean for 11 individuals (SEM))

Fig. 4 Comparison of the results obtained with the microdroplet technique for polymorphs from men with ankylosing spondylitis and from B27 negative controls. $N$-formyl-methionyl-leucyl-phenylalanine (FMLP) was used as the chemoattractant.

group of unrelated healthy B27 positive people. This PMN response was independent of disease activity, unrelated to the size of the acute phase response, and unaffected by non-steroidal anti-inflammatory drug consumption. In both this and our earlier studies $^{12}$ the PMN respnse to a chemotactic substance was increased in HLA-B27 positive men with AS. This last result, plus the normal results obtained in the B27 positive control population, make a direct association between enhanced PMN motility and HLA-B27 less likely. It is possible that a genetic factor other than B27 may be associated with the enhanced PMN response seen in B27 positive men with AS. In two separate studies involving patients with Crohn's disease ${ }^{12}$ and psoriasis vulgaris, ${ }^{13}$ however, two diseases commonly associated with the development of AS in B27 positive patients, no abnormality of PMN motility was apparent. 
Enhanced directed PMN motility is thus likely to occur as a consequence of the disease state and not as a genetically determined response. The lack of any association between PMN migration and disease activity in patients with either AS, Crohn's disease, ${ }^{12}$ or psoriasis vulgaris ${ }^{13}$ shows that PMN hypermotility is not a non-specific response common to all inflammatory disorders. Preincubation of PMNs with either AS or control sera did not enhance PMN motility: in both situations migration was mildly impaired. This impairment of motility is more likely to be due to enhanced PMN adhesion than to deactivation of PMN responsiveness to $C_{5 a}$ because PMN migration to FMLP was similarly impaired. No serum factor capable of enhancing PMN migration was detected. Enhanced PMN migration is therefore a cellular phenomenon and not a serum mediated event. In contrast with our results, Repo et al reported both a cellular and a serum abnormality in $\mathrm{B} 27$ positive individuals. ${ }^{14}$

In a uniform concentration of FMLP the PMNs from patients with AS or from controls responded in an identical fashion to all the concentrations tested (Fig. 4). Yet, under the same experimental conditions on the same 96 well plate marked differences in migration were observed in response to ZAS. Even at low concentrations of ZAS the AS PMNs were more motile than those of the controls (Fig. 3). The AS PMN response to FMLP appears to depend on the experimental technique used to measure it. When exposed to a gradient of FMLP (on the agarose plate) the AS PMN response is enhanced in comparison with the control PMN result, whereas in a uniform concentration of FMLP (as on the microdroplet plate) it is identical to the control result. One explanation for this paradox may be that the different experimental techniques measure different aspects of PMN motility. This would not explain, however, why AS PMN motility is enhanced in comparison with the control result when exposed to either a gradient or uniform concentration of ZAS. Alternatively, the differing response of AS PMNs to ZAS and FMLP may represent an abnormality at the cellular level. No single receptor on the PMN appears to be crucial in inducing this response. Enhanced PMN migration is a phenomenon which can be elicited by several different chemotactic stimuli-namely, FMLP, ZAS-or by the use of chemotactic factors liberated by live bacteria. ${ }^{12} \mathrm{An}$ investigation of the number and sensitivity of receptors on AS PMNs for $\mathrm{C}_{5 \mathrm{a}}$ or FMLP would be of interest.

In contrast with our results, Mowat and Baum did not detect any abnormality in PMN function in either 14 patients with AS or 10 with Reiter's disease. ${ }^{15}$ Similarly, Baum reported that PMN motility was normal in five patients with $\mathrm{AS}^{16}$ Both $^{\frac{0}{?}}$ these studies used the Boyden chamber technique, $\overrightarrow{\overrightarrow{\vec{D}}}$ with a long incubation period, and PMNs wereo suspended in $50 \%$ plasma as opposed to MEM. ${ }^{15}$ 16듬 These technical differences make it difficult to compare our results with theirs. Results similar tom ours have been reported in yersinia arthritis ${ }^{34}$ and Behçet's disease, ${ }^{17}{ }^{18}$ yet this phenomenon has not ${ }_{-}^{\infty}$ been reported in rheumatoid arthritis, ${ }^{15}{ }^{19}$ systemic $\vec{\circ}$ lupus erythematosus, ${ }^{20}$ or scleroderma. ${ }^{21}$ Behçet's disease, though not associated with HLA-B27, is associated with HLA-Bw51. ${ }^{22}$ An enhanced PMNa response to a chemotactic substance has beeno reported to occur as a temporary phenomenon inọ several different acute infections. ${ }^{23} 24$ The evidence $\vec{\omega}$ to support a bacterial or viral infection in theor pathogenesis of AS remains controversial, how윽 ever. ${ }^{25}$ In reactive arthritis the finding by Keat $e t$ at of chlamydial elementary bodies in synovium fromo patients with sexually acquired reactive arthritis and the persistence of $\operatorname{IgA}$ anti-yersinia antibodies in serum of patients with yersinia arthritis, ${ }^{26}{ }^{27}$ suggest that bacterial products may persist for muchoo longer than previously imagined. Investigation of PMN motility in patients with overt chronic sepsiso would be valuable. Polymorphs from patients with AS, Reiter's disease,$^{12}$ or yersinia arthritis ${ }^{3}$ demon-⿳亠二口 strate a small but definite increase in motility in̋ response to stimulation by chemotactic factors when: compared with PMNs from the control population. $\overrightarrow{\overrightarrow{0}}$ This abnormality of PMN function is not part of as generalised PMN defect because PMN adherence $\vec{P}$ phagocytosis, and superoxide production seenp unaffected. ${ }^{2} 28$ The normal results obtained for healthy B27 positive siblings of probands with ASco indicate this PMN abnormality is likely to be acquired and not 'inherited'. We could not confirm an association between PMN function and HLA. B27. The lack of any correlation between disease activity, disease severity, or the magnitude of the acute phase response and the PMN response to ther chemotactic factors implies that this phenomenon is not a consequence of an inflammatory state but an direct product of the disease process.

\section{References}

1 Pease C T, Fordham J N, Currey H L F. Increased polymorpho nuclear leucocyte (PMN) motility in B27+ve controls and patients with ankylosing spondylitis (AS). Ann Rheum Diईू 1982; $41: 313$.

2 Pease C T, Fordham J N, Currey H L F. Polymorphonuclea cell motility, ankylosing spondylitis, and HLA B27. Ann Rheunt Dis 1984; 43: 279-84.

3 Leirisalo M, Repo H, Tiilikainen A, Kosunen T U, Laitinen of Chemotaxis in yersinia arthritis. Arthritis Rheum 1980; 230 1036-43.

4 Koivuranta-Vaara P, Repo H, Leirisalo M, Kiistala U Osterman T, Vapaatalo $H$. Enhanced neutrophil migratioß 
in vivo HLA B27 positive subjects. Ann Rheum Dis 1984; 43: 181-5.

5 Repo H, Leirisalo-Repo M, Koivuranta-Vaara P. Docs enhanced neutrophil function contribute to the pathogenesis of HLA B27 associated diseases? Scand J Rheumatol /Suppl/ 1984; 52: 45-8.

6 Leirisalo M, Skylu G, Kousa M, et al. Follow-up study on patients with Reiter's disease and reactive arthritis, with special reference to HLA B27. Arthritis Rheum 1982; 25: 249-59.

7 Keat A C, Maini R N, Pegrum G D, Scott JT. The clinical features and HLA association of reactive arthritis associated with nongonococcal urethritis. $Q J$ Med 1979; 48: 323-42.

8 Khan M A, Kushner I, Braun W E. Comparison of clinical features in HLA B27 positive and negative patients with ankylosing spondylitis. Arthritis Rheum 1977; 20: 909-12.

9 Ferrante A, Thong Y H. A rapid one step procedure for purification of mononuclear and polymorphonuclear leukocytes from human blood using a modification of the Hypaque-Ficoll technique. J Immunol Methods 1978; 24: 389-93.

10 Repo H. Leukocyte migration agarose test for the assessment of human neutrophil chemotaxis. I. Effects of environmental factors on neutrophil migration under agarose. Scand J Immunol 1977; 6: 203-10.

11 McCoy J L, Dean J H, Herberman R B. Direct and indirect agarose microdroplet migration inhibition assays for detection of cell mediated immunity to tumour associated antigens. In: Bloom B R, David J R, eds. In vitro methods of cell mediated immunity. New York: Academic Press, 1976: 621-8.

12 Pease C T. Polymorphonuclear leucocyte function in ankylosing spondylitis and related disease states. London: University of London, 1987 (MD thesis.)

13 Pease C T, Fennell M, Staughton R C D, Brewerton D A. Polymorphonuclear leucocyte function in psoriasis vulgaris. Br J Dermatol 1987; 117: 161-7.

14 Repo H, Leirisalo M, Tiilikainen A, Laitinen O. Chemotaxis in yersinia arthritis. In vitro stimulation of neutrophil migration by HLA B27 positive and negative sera. Arthritis Rheum 1982; 25: 655-61.

15 Mowat A G, Baum J. Chemotaxis of polymorphonuclear leukocytes from patients with rheumatoid arthritis. J Clin Invest 1971; 50: 2541-9.

16 Baum J. Chemotaxis in human disease. In: Bellanti J A, Dayton
$\mathrm{D} \mathrm{H}$, eds. The phagocytic cell in host resistance. New York: Raven Press, 1975: 283.

17 Matsumura N, Mizushima Y. Leucocyte movement and colchicine treatment in Behçet's disease. Lancet 1975; ii: 813.

18 Takeuchi A, Kobayashi K. Mori M, Mizushima Y. The mechanism of hyperchemotaxis in Behçet's disease. J Rheumatol $1981 ; 8: 40-4$.

19 Kemp A S, Roberts-Thomson P. Neoh S H. Brown S. Inhibition of neutrophil migration by sera from patients with rheumatoid arthritis. Clin Exp Immunol 1979; 36: 423-9.

20 Perez D H, Kimberley R P, Kaplan H. Edelson H, Inman R D. Goldstein I M. Effect of high dose methylprednisolone on polymorphonuclear leukocyte function in patients with systemic lupus erythematosus. Arthritis Rheum 1981; 24: 641-7.

21 Minta J O, Kovacs E K. Sukenik S, Lee P. Chemotactic responsivemess and random motility of polymorphonuclear leukocytes from patients with progressive systemic sclerosis. J Rheumatol 1985: 12: 1125-8.

22 Ohno S, Asanuma T, Sugiura S, Wakisaka A, Aizawa M, Itakura K. HLA BW51 and Behçet's disease. JAMA 1978; 240: 529.

23 Hill H R, Gerrard J M, Hogan N A, Quie P G. Hyperactivity of neutrophil leukotactic responses during active bacterial infection. J Clin Invest 1974: 53: 996-1002.

24 Martin R R. Warr G, Couch R, Knight V. Chemotaxis of human leukocytes: responsiveness to Mycoplasma pneumoniae. J Lab Clin Med 1973; 81: 520-9.

25 Cohen S B. Ziff M. Summary of a discussion by the contributors. In: Ziff M, Cohen S B, eds. Advances in inflammation research. Vol 9. The spondyloarthropathies. New York: Raven Press, 1985: 261-4.

26 Keat A C S, Thomas B, Dixey J J, Osborn M, Sonnex C, Taylor-Robinson $\mathrm{D}$. Chlamydia trachomatis and reactive arthritis: the missing link. Lancet 1987; i: 72-4.

27 Granfor S K. Toivanen A. IgA-anti-yersinia antibodies in yersinia triggered reactive arthritis. Ann Rheum Dis 1986; 45: 561-5.

28 Repo H, Leirisalo-Repo M, Skurnik M, Tiilikainen A. Neutrophil function and HLA B27 superoxide production and yersinicidal activity of neutrophils from patients with previous arthritis. Med Biol 1984; 62: 91-4. 